\title{
AMENDMENTS
}

\section{Publisher Correction: Ultraviolet radiation-induced DNA damage is prognostic for outcome in melanoma}

Lucas D. Trucco, Piyushkumar A. Mundra, Kate Hogan, Pablo Garcia-Martinez, Amaya Viros, Amit K. Mandal, Nicolas Macagno (D), Caroline Gaudy-Marqueste, Donald Allan, Franziska Baenke, Martin Cook, Clare McManus, Berta Sanchez-Laorden (D), Nathalie Dhomen and Richard Marais (iD)

Correction to: Nature Medicine https://doi.org/10.1038/s41591-018-0265-6, published online 03 December 2018.

In the version of this article originally published, Extended Data Fig. 3 was incorrect. A duplicate of Extended Data Fig. 4 was uploaded in place of Extended Data Fig. 3. Extended Data Fig. 3 has now been uploaded. The error has been fixed in the PDF and HTML versions of this article.

Published online: 18 December 2018

https://doi.org/10.1038/s41591-018-0325-y

\section{Publisher Correction: Cannabinoid $\mathrm{CB}_{1}$ receptors in the amygdalar cholecystokinin glutamatergic afferents to nucleus accumbens modulate depressive-like behavior}

Chen-Jie Shen, Di Zheng, Ke-Xin Li, Jian-Ming Yang, Hao-Qi Pan, Xiao-Dan Yu, Jia-Yu Fu, Yi Zhu, Qi-Xin Sun,

Meng-Yu Tang, Ying Zhang, Peng Sun, Yi Xie, Shumin Duan, Hailan Hu (D) and Xiao-Ming Li (D)

Correction to: Nature Medicine https://doi.org/10.1038/s41591-018-0299-9, published online 14 January 2019.

In the version of this article originally published, there were several errors in Fig. 4. In Fig. 4a, the title read '3D repeated optical inhibition after CSDS.' It should have read '3-day repeated optical inhibition after CSDS.' In Fig. 4c, two labels that should have been aligned with the time axis appeared in the wrong place in the figure. The ticks labeled 'SI' and 'Fiber implant' should have also been labeled with ' 10 ' and '14,' respectively. Additionally, in Fig. 4j, a label that should have been aligned with the time axis appeared in the wrong place in the figure. The tick labeled 'Fiber implant' should have also been labeled with ' 14 .' The errors have been corrected in the print, PDF and HTML versions of the manuscript.

Published online: 30 January 2019

https://doi.org/10.1038/s41591-019-0372-z 